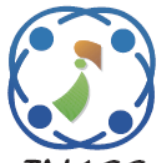

\title{
A Proposed Architecture for Pharmaceutical Supply Chain Based Semantic Blockchain
}

\author{
Shimaa Ouf ${ }^{1 *}$ \\ ${ }^{I}$ Department of Business Information Systems, Faculty of Commerce and Business Administration, \\ Helwan University, Egypt \\ * Corresponding author's Email: shimaaouf@yahoo.com
}

\begin{abstract}
This paper introduces an architecture to improve the pharmaceutical supply chain's security by using the Internet of Things, semantic web, and blockchain. This architecture increases transparency and visibility of drug flows and improves the representation of the increasing amounts of data that have been generated from pharmaceutical supply chain transactions. The pharmaceutical companies have problems dealing with the complexity of their supply chains, which represent the full life cycle of drugs from extracting raw materials, production, distribution, tracking of drugs, and quality assurance to use by patients. They need to create an efficient, effective, transparent, immutable, and secured supply chains to achieve a competitive advantage in a fast-changing market. A proposed architecture which applies the semantic web technology is implemented to enhance the representation capability of the IoT-blockchain based pharmaceutical supply chain data by annotating them with semantically rich languages to conduct formal reasoning, and aggregating data from heterogeneous sources in easy way and an interoperable manner. The proposed architecture integrates IoT, blockchain, and semantic web to help pharmaceutical companies improving their supply chains in transit and storage, improving patient satisfaction, trust through transparency, preventing drug counterfeit and sharing and reusing knowledge related to the pharmaceutical supply chain with other systems.
\end{abstract}

Keywords: Pharmaceutical supply chain, Drug delivery, Semantic web, Blockchain, IoT.

\section{Introduction}

The pharma industry provides critical health care services by supplying life-saving drugs to patients. Industrial growth relies on trusted partnerships, but fraud and cybercrime are restraining expansion. The issues of mistrust among the patients have increased because of the continuous growth of counterfeit drug supply in the market. The patients should know the full life cycle that the drugs go through to get in their hands. The life cycle of drugs includes the place where all the raw materials came from, the suppliers, the manufacturing plant where the drug was produced, and how it eventually was packaged, distributed, and ended up in the pharmacies where patients are buying it right now. This system of people, organizations, activities, resources, and information involved in moving drugs from producer to the patient is called "supply chain". There are many cases of counterfeit drugs reported worldwide, for example, Africa has a dangerous problem with counterfeit drugs in the process of transforming medication from the manufacturer to the physicians and patients [1-3].

Research studies mentioned that over 122,000 African children under the age of five lose their lives every year because of the manufacture of fake paracetamol syrup by using toxin diethylene glycol or counterfeit antimalarial. The pharmaceutical companies and the government do not disclose the cases of counterfeit drugs as it might harm their sales [4]. The World Health Organization mentioned that global counterfeited drug sales at \$75bn in 2010, a $90 \%$ increase over five years. In many developing countries in Africa, Asia, and South America, fake drugs pegged between $10 \%$ and $30 \%$ of the total medicines on sale. The growth of counterfeit drugs has pushed the regulatory authorities to trace and 
track drugs to verify its provenance as it flows across the supply chain [5].

The Internet of Things (IoT), semantic web, and blockchain will play a prominent role in the next 10 years for industrial companies as key technologies. As the price of sensors keeps falling, pharmaceutical companies will be able to overcome cost obstacles in adopting IoT technology. Applying the centralized IoT architecture by the pharmaceutical companies to trace and track the movement of drugs during the whole supply chain has several challenges; there is one authority to manage and control the collected data. Therefore, the data became easy target of various types of security and privacy attacks. IoT centralized architecture has a one point of failure in which if the server fails, the full system will be not available.

Applying blockchain technology in the pharmaceutical supply chain, will secure the data collected from the IoT because of the decentralization, immutability, and transparency of blockchain [6].

The blockchain is a decentralized technology that saves every transaction made on a network. Anyone on the network can see the content of the data for verification, no authority has been granted to a centralized company to own and operates the blockchain, and there's no opportunity to change an entry [7]. The integration of IoT and blockchain enable pharmaceutical companies to trace drugs during the entire supply chain to improve the drug quality and safety and make sure a verifiable provenance of who delivers what to whom, when, and where. These technologies are used to overcome trust issues and allowing authenticated, trustless, and secure systems of information exchange in pharmaceutical companies [8]. This ensures the provenance of drugs. Each node in the blockchain network can preview the status of where the drugs are and who has them [9].

The pharmaceutical companies which apply IoT with blockchain to trace and secure the whole supply chain and capture a large volume of data with the rapid growth of the drugs' connected things has several issues. With IoT and blockchain; there is a need to integrate semantic web to enhance the representation capability of data through knowledge management and reasoning technologies.

Besides, there is a need to promote data interoperability by improving the representation capability of the knowledge related to pharmaceutical supply chain domain-based IoT and blockchain by annotating its concepts, sub-concepts, and relations with semantically rich languages.

The semantic web technology plays a primordial role in representing the semantics of the pharmaceutical supply chain based IoT and blockchain to store the transparent and immutable transactions of the pharmaceutical supply chain.

In this paper, the theoretical foundations describe blockchain technology architecture, IoT layers, and semantic web technology. The literature review introduces the previous researches, which describes the challenges of the pharmaceutical supply chain and the impact of using blockchain to overcome these challenges.

Finally, according to the challenges of the pharmaceutical supply chain, a proposed architecture has been introduced to improve the pharmaceutical supply chain by merging IoT, blockchain, and semantic web technologies.

\section{Theoretical foundations}

This section introduces the challenges of the pharmaceutical supply chain and the three technologies, which play an important role in this paper: 1) Internet of Things (IoT), 2) blockchain, and 3) semantic web.

\subsection{Pharmaceutical supply chain management}

The supply chain is defined as the end-to-end system that represents the life cycle of drugs, which includes planning, information source, inventory, production, location, transportation, delivery, the return of a product and issue a refund to satisfy the patient requirements as efficiently as possible. The pharmaceutical supply chain covers:

Ingredients: include natural resources (water and fresh herbs) to produce drugs.

- Production: includes the process of creating the Final product (drugs) by bringing all the components together.

- Retail: after the drugs have been created, they need to be shipped to pharmacies.

- Logistics and Warehousing: they will help in getting ingredients, materials, and finished drugs to the right place at the right time to maintain the pharmaceutical supply chain operating efficiently.

- Customer: he buys the product either from the pharmaceutical companies or from a pharmacy.

- Return: a successful pharmaceutical supply chain should not be unidirectional. It should move back.

- If the drugs do not satisfy the patient's needs, he can return it and get a refund $[10,11]$.

In recent years, the process of tracing the entire pharmaceutical supply chain life cycle becomes very complicated. The components of drugs come from different sources. The patient does not know the 
source where the defective drugs come from, which leads to a lack of security and transparency.

\subsection{Internet of things (IoT)}

It connects the physical world with the digital world through IoT technology. This technology is used to:

1. Gather data from real-world objects (drugs).

2. Make the processing of data to produce information.

IoT provides users or systems with accurate information to help them make the right decisions [12]. The IoT architecture encompasses a set of layers:

Sensing layer (Things): refers to the devices (like sensors) attached to drugs to gather and communicate data related to their identity, Geo-location, and behavior and detect changes in the environment. The data has been transferred to the other layers by sensors.

Network layer: is used to send the collected data from the sensors to the internet. The process of sending information from the sensing layer to the augmented intelligence layer has been supported by the protocols, hardware, and software [13].

Augmented intelligence: analyze raw data collected from the sensing layer to make decisions. Application Layer: is a user-centric layer that is used to preview the results of the augmented intelligence layer [14-15].

\subsection{Blockchain architecture}

The blockchain architecture is composed of several components and has important properties for the pharmaceutical supply chain:

The Block: A blockchain is composed of a chain of a sequence of blocks. Each new block is connected to the previous block to store data related to the specific domain. For Example, A drug block contains data about the drugs, Name, Type, Amount, and Ingredients.

Hash: Each block in the chain must have a hash that is used to identify a block and all of its components. It is considered a fingerprint for each

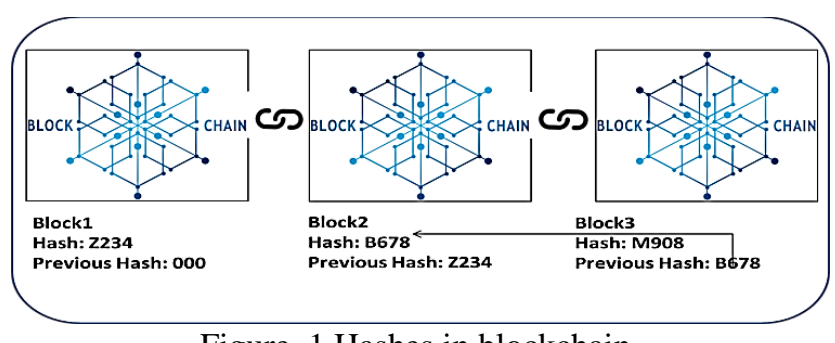

Figure. 1 Hashes in blockchain

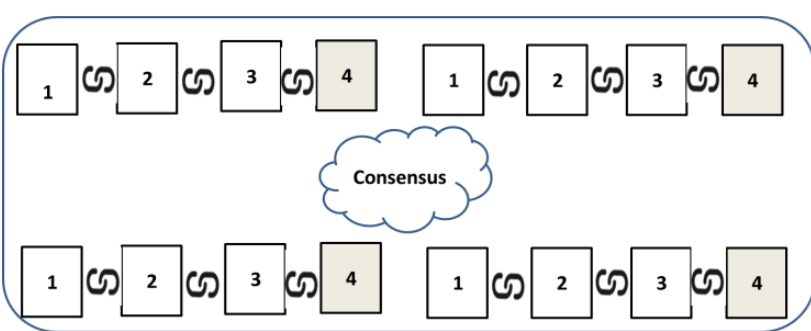

Figure. 2 Distributed peer-to-peer network

Proof of Work: The concept of Proof-of-Work is used to make blockchain more secure and prevent tampering through including complex cryptography that takes time and effort to solve [14-15].

Decentralized ledger: The pharmaceutical company has complete controls on data and we cannot ensure $100 \%$ that the data that pharmaceutical companies are introduced is $100 \%$ authentic or not. Blockchain technology plays a crucial role in solving the problem of data isolation. Blockchain technology is used as a decentralized ledger as shown in Fig. 2. Each participant in the network shares data stored in the blockchain network and one centralized person does not own it.

When a participant (node) creates a new block, all participants in the chain will receive this new block. Each participant in the chain must validate each block and then a new block has been added to the blockchain. A consensus determines the validity of each block and rejects the tampered blocks [14].

\subsection{Semantic web}

The semantic web technology is the future of the pharmaceutical supply chain. One of the most popular and acceptable definitions of ontology is Gruber's: "ontology is an explicit specification of a conceptualization" [16].

Ontologies are used to represent knowledge about the IoT-blockchain based pharmaceutical supply chain. Ontologies support the process of sharing and reusing the data that is generated from IoT devices. The blockchain technology is represented as a peerto-peer network, in which different participants, roles, assets, interfaces, and transactions are interconnected and interacted with each other. Ontologies are well suited to represent the activities within blockchainbased pharmaceutical supply chain transactions [17].

Blockchain is considered a perfect complement to ontology to configure a big part of Tim Berners-Lee's vision of the semantic Web. Therefore, blockchain and ontologies must co-evolve to achieve this complementarity. One area of investigation has been in representing a blockchain technology and components using ontologies. This paper develops 
ontology of pharmacy supply chain based blockchain as a conceptual modeling formalism.

\section{Research method}

In recent years, IoT, blockchain, semantic web and play a crucial role in scientific research. There is much academic researches that discussed the impact of the IoT, blockchain, and semantic web play to secure and improve the pharmaceutical supply chain ecosystem. The research papers' classification on IoT and blockchain will be verified by their publication scientific database. We searched the following leading scientific databases to achieve a full bibliography of research papers on IoT, blockchain, and semantic web and their crucial role to increase the accuracy and effectiveness of the pharmaceutical supply chain: ScienceDirect; Springer; and IEEE Library Link. We performed the search based on three keywords: "the role of IoT and blockchain in counterfeit drugs", "blockchain and ontologies in the pharmaceutical supply chain", and "Pharmaceutical supply chain risks". The search results about IoT, semantic web, and blockchain and their leading role in the pharmaceutical supply chain was 274 research papers selected from the leading scientific databases. The scientific papers that were truly related to our paper, are going to be explained. All the retrieved papers were reviewed whereby similar papers were ignored followed by selection based on abstract and finally based on the full-text reading.

\subsection{Classification by scientific databases}

The scientific papers are categorized according to scientific databases as introduced in Table 1. IEEE introduced over $74 \%$ (204 out of 274 research papers or $74.5 \%)$ of the total number of papers. ScienceDirect (50 papers out of 274 research or $18.2 \%$ ) and Springer Link (20 out of 274 research papers, or $7.3 \%$ ), introduced the second and third largest percentage papers on the role of IoT, semantic web, and blockchain in the pharmaceutical supply chain among the scientific databases.

All research papers are chosen from three different scientific databases, 274 papers were found using selected keywords based on title searching.

Table 1. Categorizing research papers by scientific databases

\begin{tabular}{|c|c|c|}
\hline $\begin{array}{c}\text { Scientific } \\
\text { databases }\end{array}$ & Amount & Percentage \\
\hline IEEE Library & 204 & $74.5 \%$ \\
\hline ScienceDirect & 50 & $18.2 \%$ \\
\hline SpringerLink & 20 & $7.3 \%$ \\
\hline Total & 274 & $100 \%$ \\
\hline
\end{tabular}

After that, duplicate papers were checked and deleted, leaving only 204 papers. The screening process continued by eliminating paper that did not meet selection criteria based on abstract review resulting in 70 selected papers. Finally, 20 selected papers were used for this paper after 50 papers were eliminated because the papers include an insufficient discussion on how IoT, semantic web, and blockchain enhance the pharmaceutical supply chain. All papers address the shortcoming in the pharmaceutical supply chain and how IoT, semantic web, and the blockchain is used for enhancing the pharmaceutical supply chain. Researchers in these papers ignored integrating three technologies (IoT, semantic web, and blockchain). In this paper, we represent a proposed paradigm to achieve an effective, efficient, immutable, and transparent pharmaceutical supply chain by integrating the three technologies (IoT, semantic web, and blockchain).

Table 2. Comparison between the research papers

\begin{tabular}{|c|c|c|c|c|}
\hline \multirow[t]{2}{*}{ Authors/Technology } & \multicolumn{2}{|c|}{$\begin{array}{l}\text { Semantic } \\
\text { Web }\end{array}$} & \multirow[b]{2}{*}{ 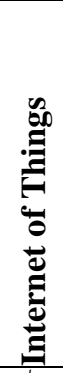 } & \multirow[b]{2}{*}{ 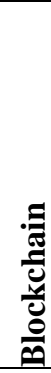 } \\
\hline & 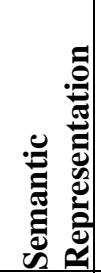 & 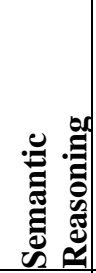 & & \\
\hline (Liu and Jia 2010) [18] & $x$ & $x$ & $\sqrt{ }$ & $x$ \\
\hline $\begin{array}{l}\text { (Altunkan, Yasemin et al. } \\
\text { 2012) [19] }\end{array}$ & $x$ & $x$ & $\sqrt{ }$ & $x$ \\
\hline (Mettler 2016) [20] & $x$ & $x$ & $x$ & $\sqrt{ }$ \\
\hline $\begin{array}{l}\text { (Bahga and Madisetti 2016) } \\
\text { [14] }\end{array}$ & $x$ & $x$ & $x$ & $\sqrt{ }$ \\
\hline $\begin{array}{l}\text { (Alangot and Achuthan } \\
\text { 2017) }[21]\end{array}$ & $x$ & $x$ & $\sqrt{ }$ & $\sqrt{ }$ \\
\hline (Kuzmin 2017) [29] & $x$ & $x$ & $\sqrt{ }$ & $\sqrt{ }$ \\
\hline $\begin{array}{l}\text { (Bocek, Rodrigues et al. } \\
\text { 2017) [22] }\end{array}$ & $x$ & $x$ & $\sqrt{ }$ & $\sqrt{ }$ \\
\hline $\begin{array}{l}\text { (Chamekh, El Asmi et al. } \\
\text { 2017) [23] }\end{array}$ & $x$ & $x$ & $\sqrt{ }$ & $x$ \\
\hline (Wazid, Das et al. 2017) [24] & $x$ & $x$ & $\sqrt{ }$ & $x$ \\
\hline $\begin{array}{l}\text { (Trokanas and Srai 2017) } \\
\text { [25] }\end{array}$ & $\sqrt{ }$ & $x$ & $x$ & $x$ \\
\hline $\begin{array}{l}\text { (Boulos, Wilson et al. 2018) } \\
\text { [26] }\end{array}$ & $x$ & $x$ & $x$ & $\sqrt{ }$ \\
\hline $\begin{array}{l}\text { (Westerkamp, Victor et al. } \\
2018,2019) \text { [27][30] }\end{array}$ & $x$ & $x$ & $x$ & $\sqrt{ }$ \\
\hline $\begin{array}{l}\text { (Seebacher and Maleshkova } \\
\text { 2018)[17] }\end{array}$ & $\sqrt{ }$ & $x$ & $x$ & $\sqrt{ }$ \\
\hline (Zhou, Wang et al. 2018) [9] & $x$ & $x$ & $x$ & $\sqrt{ }$ \\
\hline (Miller 2018) [6] & $x$ & $x$ & $\sqrt{ }$ & $\sqrt{ }$ \\
\hline $\begin{array}{l}\text { (Dujak and Sajter 2019) } \\
\text { [28] }\end{array}$ & $x$ & $x$ & $x$ & $\sqrt{ }$ \\
\hline
\end{tabular}




\section{Literature review}

The authors propose an e3-value methodology to track and trace drugs using IoT technology. This methodology mentioned that the IoT application creates the value of the drug supply chain by facilitating information sharing, helping manufacturers optimizing inventory, reducing operating costs, and introducing an accurate supply chain. The authors of this paper have created the value-based business model of IoT applied in the drug supply chain [18]. In this paper, IoT helps pharmaceutical companies to optimize inventory and providing synchronous supply precisely. However, this paper ignored applying blockchain technology to prevent tamper data and semantic web to manage a continuously huge amount of generating data from the connected sensors.

The authors address the important role that IoT plays in Turkish Pharmaceuticals Track and Trace Systems (ITS). ITS secures health and wealth by tracking pharmaceuticals in the supply chain to ensure a reliable supply of drugs to patients. This paper ignored the important role which blockchain plays to ensure a decentralized, immutable, and transparent pharmaceutical supply chain. Besides, it ignored the important role which semantic web play to manage a large amount of generating data from the IoT devices [19].

The author shows that blockchain technology becomes the beginning to change the services related to the healthcare sector, such as user-oriented medical research, public healthcare management, and drug counterfeiting in the medical sector. In this paper, the authors address the goals, influences, and potential connection to this technology. They mentioned that the blockchain promotes a new digital business model and digital health initiatives and strongly affects the balance of power between existing market players in healthcare. It eliminates intermediaries and opens new doors concerning how market interactions in healthcare can be conducted. It fights counterfeit drugs in the pharmaceutical industry, whereas drugs are associated with a sensitive production process and where extensive liability and reputational issues are related to the final drugs [20].

Because of increasing counterfeit drugs and the existence of substandard drugs, which lead to loss of life, merging IoT framework with blockchain to manage and communicate the exchange and flow of data between trusted parties is proposed in this paper. A proposed framework for tracking the pharmaceutical supply chain is introduced to prove the safety of drugs as it travels through the supply chain. The blockchain technology prevents the existence of counterfeit drugs and ensures an immutable record of drugs [21].

In the medical industry, ensuring quality control and regulatory compliance over the drugs' transportation requires many complexes and strict environmental control processes (humidity and temperature). Therefore, the authors propose modum.io, which depends on IoT sensor devices leveraging blockchain technology to maintain public accessibility of temperature records and data immutability, while decreasing operational costs in the pharmaceutical supply chain. During the shipment, the sensor devices monitor and capture the temperature of each parcel. All data is transferred to and securely stored in the blockchain [22].

The authors explain the impact of using the internet of things to improve inventory management in the pharmaceutical supply chain by tracking drugs to prevent harmful drugs. Pharmaceutical companies need to improve the quality of drugs' distribution. Context-aware middleware for RFID based pharmaceutical supply chain is proposed to offer a deeper intelligence for the objects monitoring and handling frequent changes and risks in pharmaceutical logistics by applying IoT and the semantic web [23]. This paper ignored the crucial role which blockchain plays to improve the accuracy of the pharmaceutical supply chain.

In this paper, authors use IoT technologies to propose a solution to examine medicine-related problems. They use the Internet of Things to create an authentication scheme for preventing drug counterfeiting. This scheme is used to check the authenticity of drugs and ensure an effective supply chain [24]. This paper ignored to Integrate blockchain and ontologies with IoT to ensure the immutable and transparent supply chain and to manage a huge amount of data in an efficient manner.

The authors propose an ontological model to represent knowledge, which related to the pharmaceutical supply chain domain. The proposed ontological model is used to enhance transparency and traceability of drugs by its ability to query all systems in the supply chain network. The authors validate their work by applying the proposed framework on pharmaceutical companies in the UK. The proposed framework has a powerful role to facilitate future implementations and extensions of the system to enable ad hoc supply chain configuration by using the semantic web [25]. This paper does not focus on integrating IoT and blockchain with semantic web to improve the security and transparency of the pharmaceutical supply chain. 
The authors in this paper show the vital role that blockchain plays in medical and healthcare research. They reflect that blockchain technology is being investigated for public health and healthcare in different ways. Blockchain becomes a strong competitor in restructuring, the healthcare landscape of the world abroad since its foundations of cryptographic security, decentralization, and immutability. The authors of this paper, explore the role that blockchain technology plays to improve supply chain management in the pharmaceutical companies including eliminating or reducing fraud and errors, minimizing costs of the courier, managing inventory, and increasing customer and trust identifying. Blockchain technology can be applied in the pharmaceutical supply chain by attaching unique device identifiers for each medical device and by keeping track and issuing firmware updates by using smart contracts [26].

The authors represent the limitations of using centralized systems, which cause suffering from isolated data storage and lacking trust when multiple parties are involved. Blockchain technology comes to solve these issues by enabling tracking across multiple entities. The authors propose a model for tracing the supply chain and product transformations based on blockchain technology. Ethereum Virtual Machine [27] implements the proposed system.

The paper introduces the leading role that blockchain plays in securing logistics and supply chain networks. The new implementations of supply chain based on blockchain ensure the secure exchange of information and storage, automatization of a transaction, overcoming trust shortcoming, ensuring authenticated and trustless logistics [28].

Based on the related works and as mentioned in Table 2, we found that researchers ignore merging IoT, semantic web, and blockchain technologies to improve the effectiveness of the pharmaceutical supply chain. As a result, this paper proposed an enhanced paradigm for improving the pharmaceutical supply chain by merging IoT, semantic web, and blockchain technologies.

\section{A proposed architecture of pharmaceutical supply chain based semantic blockchain (PSCSB)}

Wherever Times is specified, Times Roman of Times New Roman may be used. If neither is available on your word processor, please use the font closest in appearance to Times. Avoid using bitmapped fonts if possible. True-Type 1 fonts are preferred.
In this section, we describe A Proposed Architecture of Pharmaceutical Supply Chain based Semantic Blockchain (PSCSB) that is used for securing the pharmaceutical supply chain. The IoT, blockchain, and semantic web technologies are considered the backbone and a key driver for the pharmaceutical supply chain. These technologies enable transparent, reliable, and real-time information, trust among participants through providing immutable and tamper-proof records of transactions and ensure better supply chain provenance. The proposed architecture of the semantic blockchain pharma supply chain composed of three layers as shown in Fig. 3.

\subsection{Layer 1: internet of things}

This layer represents the hardware layer and comprises wireless sensor networks and Radio Frequency Identification (RFID). These things are integrated into the whole drugs' life cycle and can be supervised from any location at any time. Radio Frequency Identification is used to check the object's location while wireless sensor networks are used to monitor and measure environmental conditions. Sensors collect data from the object under measurement in the pharmaceutical supply chain and turn it into useful information to make a decision. IoT technology is used to increase the overall performance of the pharmaceutical supply chain because of its ability to minimize waste and address real-time issues instantaneously.

\subsection{Layer 2: semantic web}

It represents knowledge related to the whole components, relationships, and transactions of the IoT blockchain-based pharmaceutical supply chain domain. The semantic representations of all the components of PSCSB are represented in this layer. The ontology for representing all the transactions related to IoT blockchain-based pharmaceutical supply chain domain is created and implemented from scratch to achieve research objectives (reliable, immutable, secured, efficient, and effective pharmaceutical supply chain ecosystem). This ontology is represented using Web Ontology Language Description Logic (OWL-DL). ProtégéOWL is used to create this ontology.

The evolution of technology introduces new solutions for improving the pharmaceutical supply chain and makes it easier and effective. Ontologies enable the formal and explicit representation of all concepts and properties (object properties and datatype properties) in a way that it can be shared and reused by many pharmaceutical supply chain systems. 
Ontologies support the extraction of new knowledge by applying inference mechanisms on the knowledge represented in the ontology. The components of the semantic layer are blockchain network and pharmaceutical supply chain transactions.

IoT devices store all the generated data on a centralized system, which effects on data security, privacy, and safety; data are vulnerable to losing, hacking, and manipulation. Blockchain comes to overcome IoT challenges due to the decentralized, autonomous, immutable, transparent, and trusted capabilities of blockchain.

Blockchain technologies are built on a peer-topeer network, where different interfaces, participants, assets, and transactions are interconnected with each other. Blockchain is used in the pharmaceutical supply chain to improve the tracking, transparency, and privacy issues using an immutable record of data, distributed and decentralized storage, and controlled user access.

IoT devices collect data, which are related to pharmaceutical supply chain transactions from different sources, and these data are relayed to the semantic blockchain based-pharmaceutical supply chain for making processing and storing.

The pharmaceutical supply chain based semantic blockchain would capture key data emitted from IoT devices to trace the drugs' origin and its transformation process. The captured transactions reflect the pharmaceutical supply chain's life cycle. Which contains the process of extracting natural resources (raw materials), manufacturing, which include converting the natural resources into finished drugs, distributing the finished drugs ready to sell takes place. The transactions are authenticated and time-stamped at each step to ensure transparent traceability through the production and distribution channels. This can add value to the finished drugs by preventing the drugs from falling into the wrong hands and authenticating the drug for the patient that reduces the price manipulation and delivery of invalid drugs. The correctness of the transactions is validated by a consensus mechanism to generate the block.

The smart contracts as the core component in the blockchain are used to represent a set of actions to decide if transactions are valid by applying business rules. These rules are used to describe the role of the pharmaceutical supply chain's participants. The participants of the pharmaceutical supply chain can collaborate and communicate as follow:

The first step represents the natural resources as the raw material; the suppliers of the natural resources are submitting transactions on the pharmaceutical supply chain ontology. These transactions contain raw material ID, name, quality, quantity, description, and origin Geo-location.

The second step includes manufacturing raw materials to produce the final drugs; the appropriate transactions are submitted into supply chain ontology. These transactions contain manufacturer name, experience, date and time, and location.

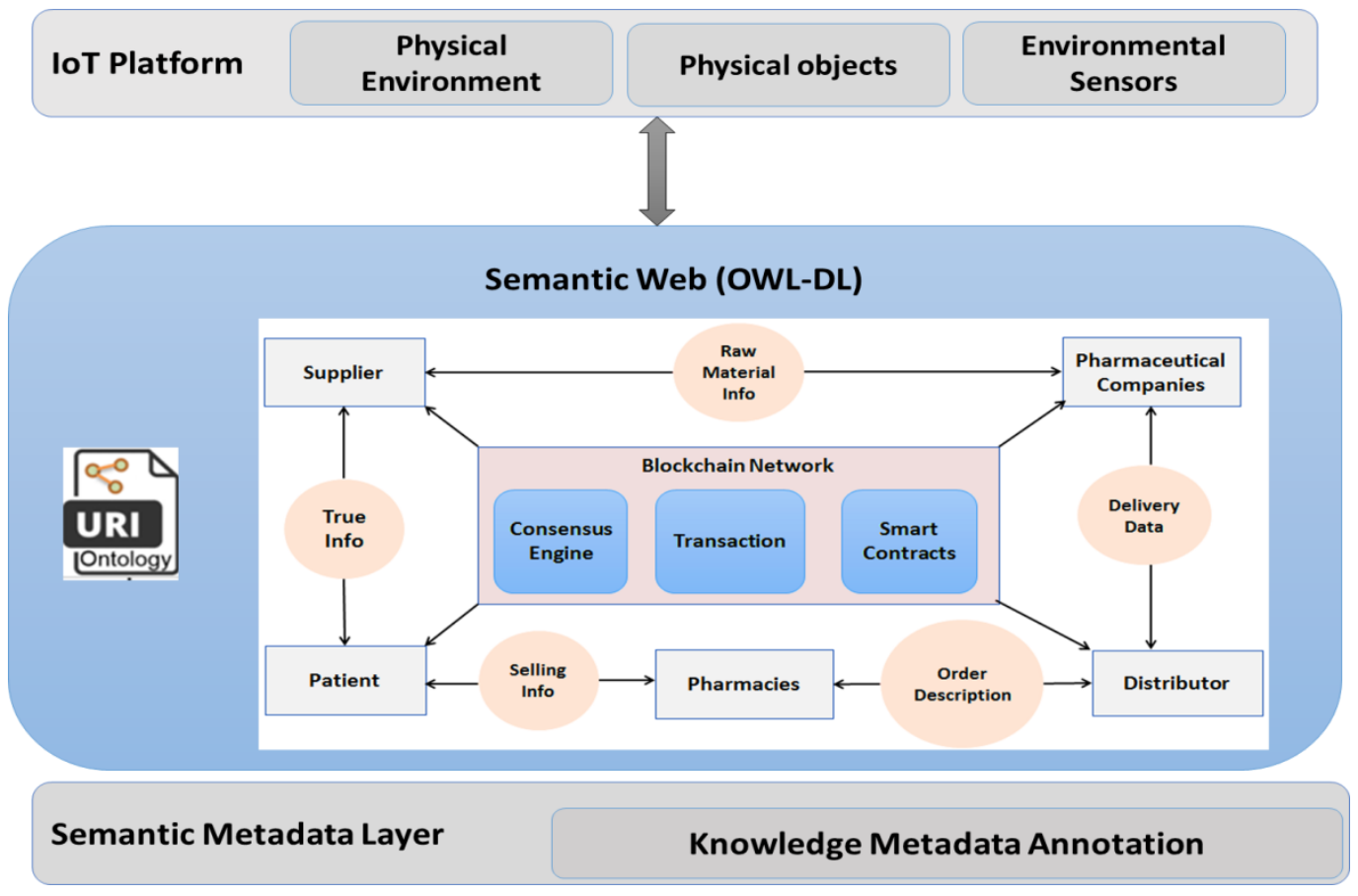

Figure. 3 The proposed architecture 
In the third step, the finished drugs are handed to the distributors. The distributors sell the finished drugs to pharmacies (retailers). These transactions contain important data, such as the distributor name, pharmaceutical company name, pharmacy name, Geo-location, address, quantity, details, and quality.

Next, moving the finished drugs from source to destination is recorded in the supply chain (container location, and timestamp). The last step represents the process of selling drugs to the patient. As a result, the following five participants are included in the blockchain-based pharmaceutical supply chain:

Producers: the pharmaceutical companies are responsible for producing the final drugs.

Distributor: is an intermediate entity between the pharmaceutical companies and pharmacies.

Pharmacies: represent retailers who responsible for selling the drugs to patients;

Patient: the final participant of the chain who buys the drugs.

Endorser: receives a transaction in the form of a transaction proposal. Endorser checks transactions to prevent suspicious transactions from passing through the network.

\subsection{Layer 3: semantic metadata}

This layer annotates all the concepts, subconcepts, and their properties (objects and data types), which represent the pharmaceutical supply chain based semantic blockchain (metadata-description of knowledge representation).

\section{Implementing a prototype of the proposed architecture (PSCSB)}

We develop the proposed architecture from scratch using protégé 5 .

Knowledge gathering is an important requirement for developing the ontology because it presents how the knowledge of the pharmaceutical supply chain domain is gathered. In this paper, as a result of considering the pharmaceutical supply chain phases, and blockchain components, the data were gathered mainly from the research papers which discuss the components and phases of pharmaceutical supply chain. As well as, interviewing a group of the domain experts in the pharmaceutical companies was carried out.

All the required concepts and sub-concepts, object properties, data properties, and the relations between concepts and sub-concepts and the properties of these relations related to the pharmaceutical supply chain domain are represented in Figs. 4, 5, and 6. Four steps are applied to develop ontology: step1 generate the conceptual representation of the proposed pharmaceutical supply chain, step 2 creates concepts and sub-concepts, step 3 defines object properties, and step 4 defines datatype properties.

STEP 1: The proposed pharmaceutical supply chain based semantic blockchain includes six categories that explain the components of the pharmaceutical supply chain. These components include Block, HashBlock, Blockchain, Verification, Transaction, and Node as shown in Fig. 7. The pharmaceutical supply chain based semantic blockchain composed of a set of nodes (participants). A transaction is generated by a node and verified by other participating nodes in the blockchain. The transaction class is responsible for creating the transaction and includes the sender ID, recipient ID, and details. Each transaction should be verified. Verification class responsible for verifying the transaction before being included in a block and verifying the block before being added to the blockchain. The hash block class responsible for generating hash string consists of 256 bits for each block. The block class contains a unique index for each block, previous block's hash, timestamp, and transactions that are included in a block and the generated proof from the verification class. The block class responsible for creating a block through aggregating sets of verified transactions that can form a block and generating a hash from hash block class.

STEP 2: All concepts and sub-concepts that related to a pharmaceutical supply chain based semantic blockchain domain are shown in Table 3 and Fig 8. The whole life cycle of the pharmaceutical supply chain is represented by the concepts and sub-concepts.

OWL vocabulary is used to represent all the concepts and sub concepts. The following OWL code shows an excerpt of these concepts:

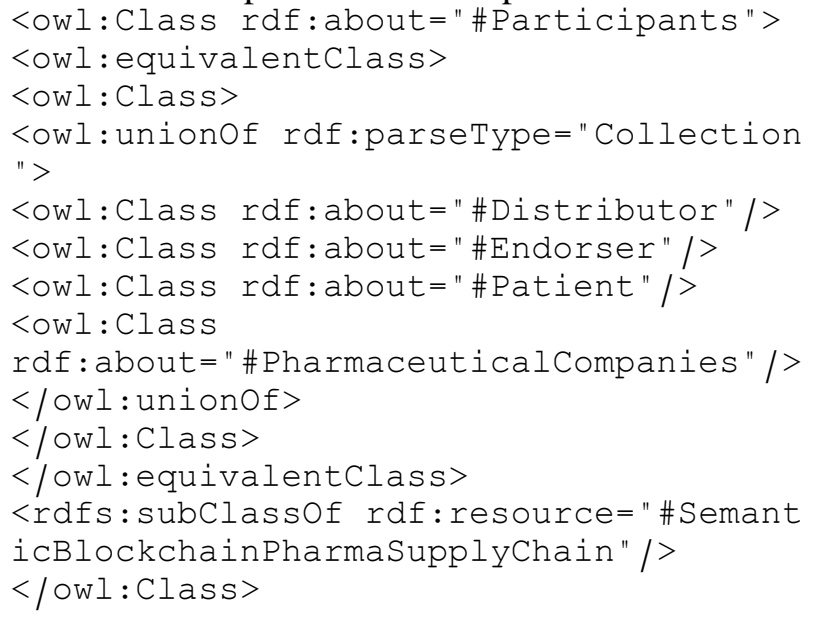

This OWL concept hierarchy shows that Participants concept (which considered unionOf Distributor, Endorser, Patient, 
PharmaceuticalCompanies)

is subClassOf.SemanticBlockchainPharmaSupplyChai n concept.

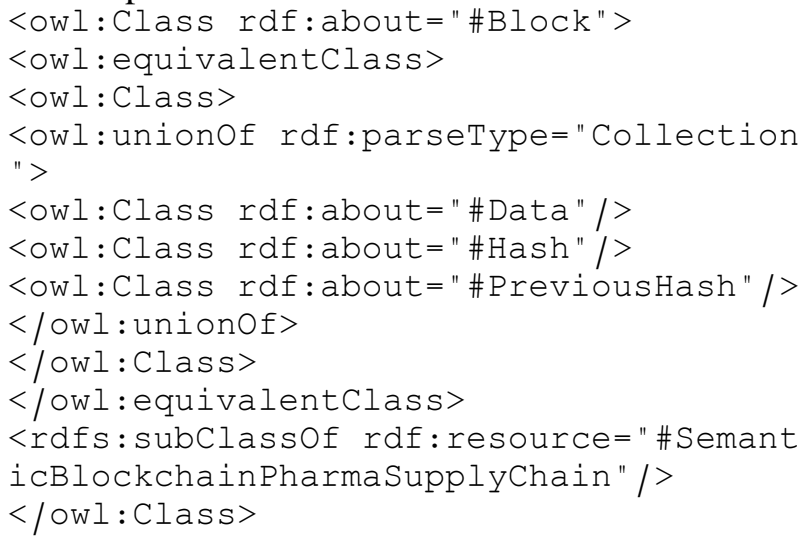

This OWL concept hierarchy shows that Block concept (which considered unionOf Data, Hash, PreviousHash) subClassOf.SemanticBlockchainPharmaSupplyChai n concept.

STEP 3: Represents object properties of the pharmaceutical supply chain. Describing the object properties is based on the relationship, which the author wants to add between concepts and subconcepts of the pharmaceutical supply chain. These relationships are represented using domain and range using OWL as shown in the following:

<owl: ObjectProperty rdf:about=" \#isPati ent" $>$

<rdfs:range rdf:resource=" \#Patient " / >

$<$ rdfs: domain>

<owl: Class>

<owl:unionof rdf:parseType="Collection $">$

<owl: Class rdf:resource=" \#Participants $" />$

<owl: Class rdf:resource="\#SemanticBloc kchainPharmaSupplychain " /

$</$ owl : unionof>

$<$ owl: Class $>$

$<$ rdfs: domain>

$</$ owl: ObjectProperty $>$

The OWL object property isPatient represents the relationship between the unionOf of Participants,

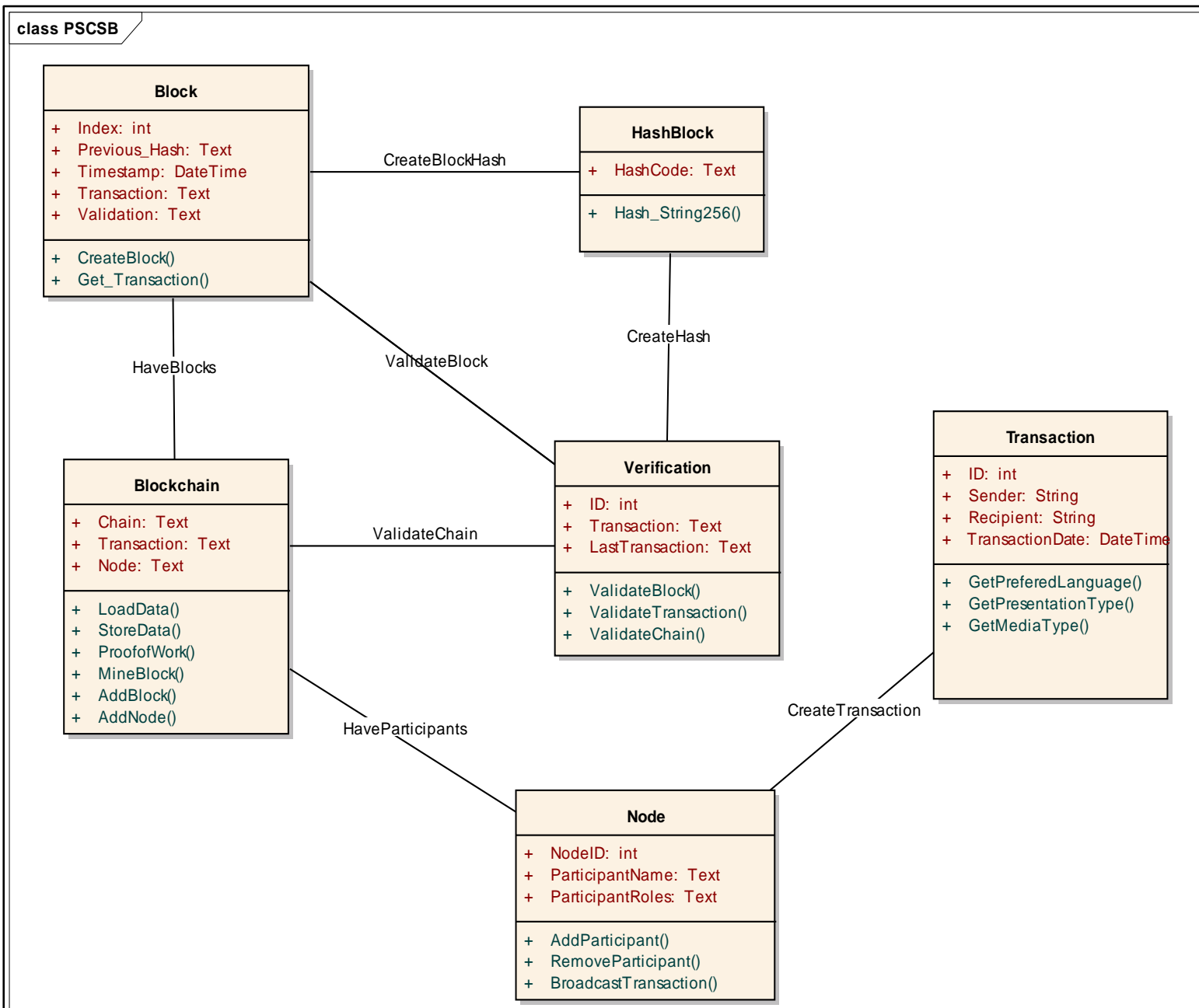

Figure. 4 The conceptual representation of the pharmaceutical supply chain 


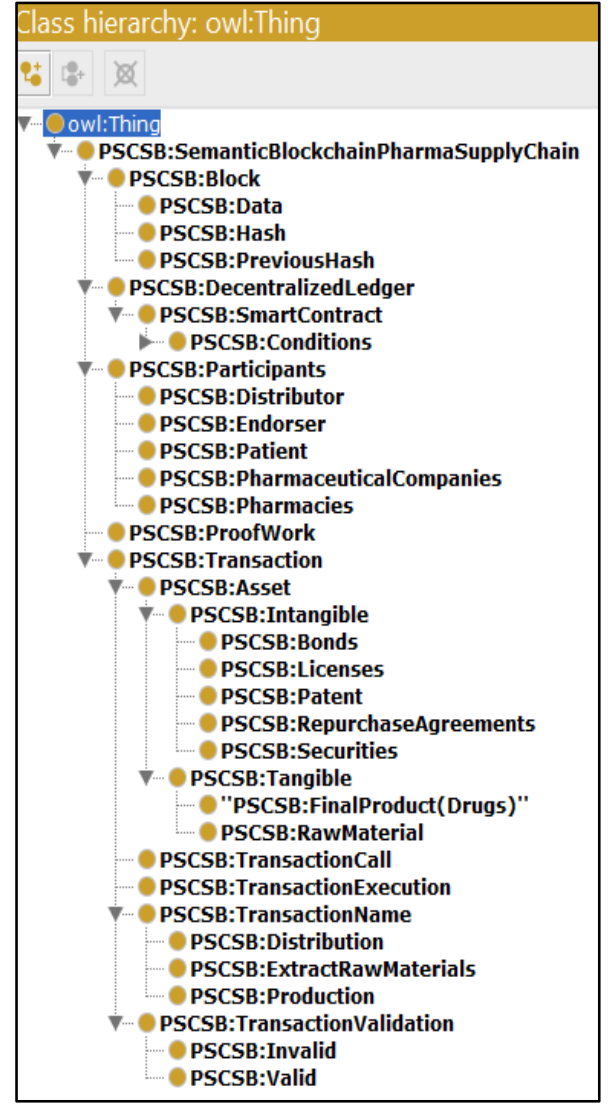

Figure. 5 An excerpt of pharmaceutical supply chain based semantic blockchain

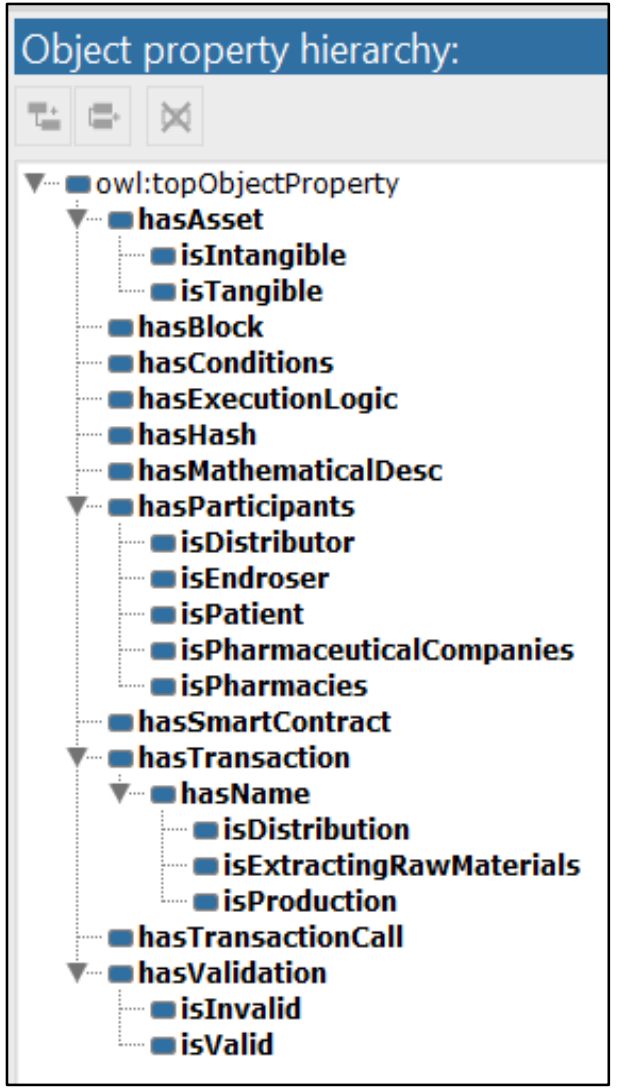

Figure. 6 The object properties among pharmaceutical supply chain based semantic blockchain classes

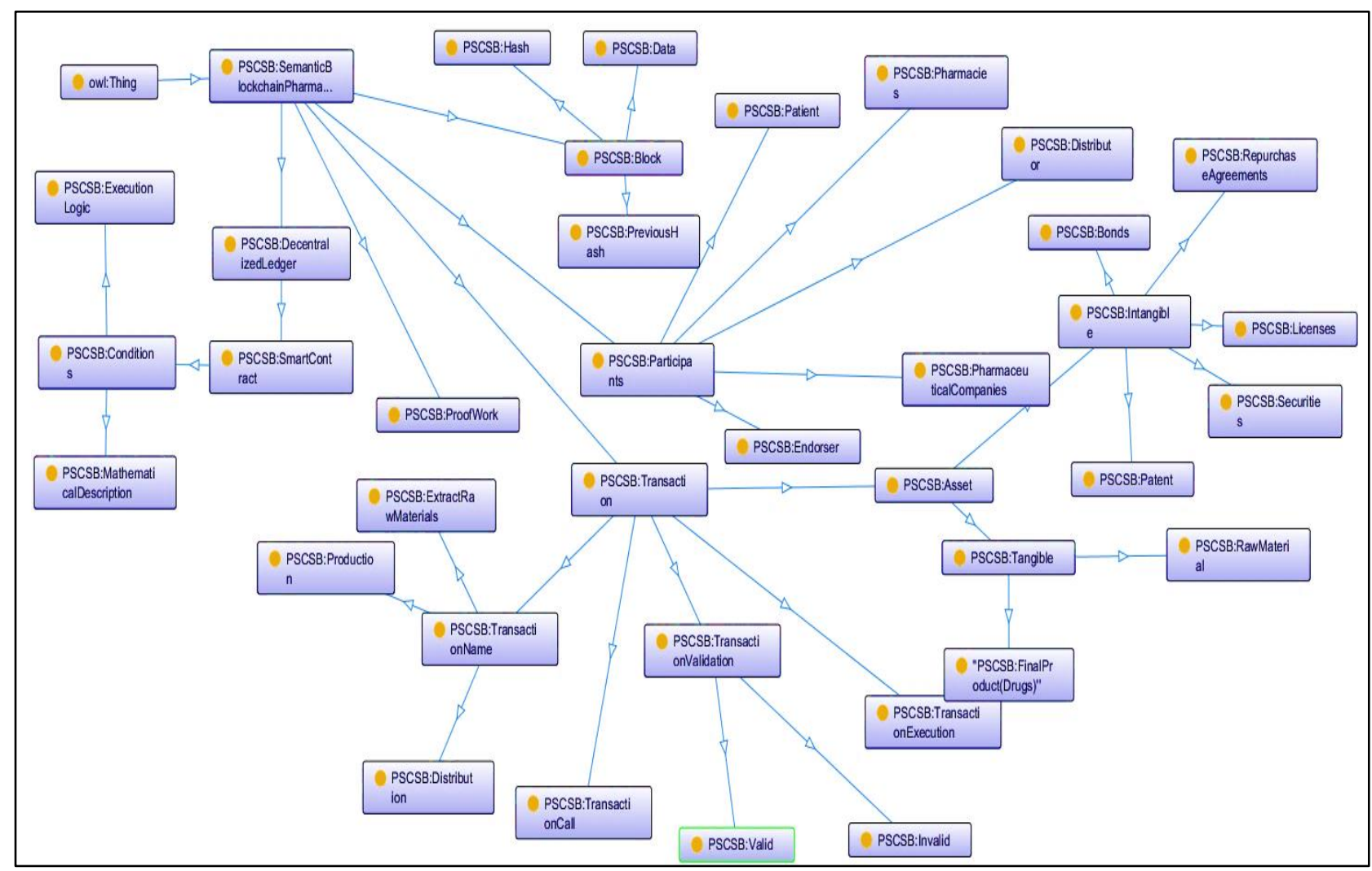

Figure. 7 An excerpt of pharmaceutical supply chain based semantic blockchain using OWL-VIZ 
SemanticBlockchainPharmaSupplyChain concepts, which represent the domain, and the Patient concept, which represents the range.

<owl: ObjectProperty rdf: about=" \#hasBlo $\mathrm{ck} ">$

<rdfs:domain rdf:resource=" \#SemanticBl ockchainPharmaSupplyChain" />

$<$ rdfs: range rdf:resource=" \#Block" | > </owl: ObjectProperty>

The OWL object property hasBlock represents the relationship between the SemanticBlockchainPharmaSupplyChain concept, which represents the domain, and the Block concept, which represents the range.

STEP-4 Create Data Type Properties: As shown in the following part, the relationship between an individual and a primitive data type are defined by data type properties.

OWL-Viz is used to visualize the pharmaceutical supply chain based semantic blockchain as shown in Fig. 7.

\section{Conclusion and future work}

The semantic web is used to promote information interoperability by improving the representation capability of supply chains and blockchain components by annotating them with semantically rich languages. It facilitates a shared understanding between humans about IoT- blockchain-based pharmaceutical supply chain. Ontology is created from scratch to represent the knowledge related to the blockchain-based pharmaceutical supply chain to be shared and reused in other pharmaceutical supply chain management systems. Using these technologies in the proposed architecture has allowed providing immutable distributed storage for tracing and tracking drugs. In the future, we plan to implement the other layers of the proposed architecture to realize and evaluate our approach. The proposed Architecture of Pharmaceutical Supply Chain based Semantic Blockchain (PSCSB) is flexible to integrate different technologies like IoT, blockchain, and semantic web. This integration secures pharmaceutical supply chain and provides an efficient mechanism to track drugs and detect any suspicious activity. It provides a decentralized architecture and facilitates sharing and reusing components of pharmaceutical supply chain.

Extra researches will be assigned to apply the Semantic Web Rules and SPARQL queries to infer new knowledge from the Pharmaceutical Supply Chain based Semantic Blockchain (PSCSB).

\section{References}

[1] S. E. D. Nsimba, "Problems associated with substandard and counterfeit drugs in developing countries: A review article on global implications of counterfeit drugs in the era of anti-retroviral (ARVS) drugs in a free market economy", East African Public Health Association Vol. 5, No. 3, pp. 205-210, 2009.

[2] E. Blackstone, J. Fuhr, and S. Pociask, "The health and economic effects of counterfeit drugs", American Health \& Drug Benefits, Vol. 7, No. 4, pp. 216-224, 2014.

[3] G. Nayyar, J. Breman, Tim. Mackey, J. Clark, M. Hajjou, M. Littrell, and J. Herrington, "Falsified and substandard drugs: Stopping the pandemic", The American Journal of Tropical Medicine and Hygiene, Vol. 100, No. .5, pp. 1058-1065, 2019.

[4] T. Ahram, A. Sargolzaei, S. Sargolzaei, J. Daniels, and B. Amaba, "Blockchain technology innovations", In: Proc. of Technology \& Engineering Management Conf. (TEMSCON), pp. 137-141, 2017.

[5] Z. Huang, X. Su, Y. Zhang, Ch. Shi, H. Zhang, and L. Xie, "A decentralized solution for IoT data trusted exchange based-on blockchain", In: Proc. of Computer and Communications (ICCC), 3rd IEEE International Conf. on Computer and Communications (ICCC), pp. 1180-1184, 2017.

[6] D. Miller, "Blockchain and the Internet of Things in the Industrial Sector", IT Professional, Vol. 20, No. 3, pp. 15-18,2018.

[7] H. Jiang, H. Peng, and S. Dian, "A Design of Medical Information Sharing Model Based on Blockchain Technology", In: Proc. of IOP Conf. Series: Materials Science and Engineering, IOP Publishing, Vol. 428, No. 1. 2018.

[8] Y. Rahulamathavan, R. Phan, M. Rajarajan, S. Misra, and A. Kondoz, "Privacypreserving blockchain based IoT ecosystem using attributebased encryption", In: Proc. of International Conf. on Advanced Networks and Telecommunications Systems (ANTS), pp. 1-6, 2017.

[9] L. Zhou, L. Wang, Y. Sun, and P. Lv, "BeeKeeper: A Blockchain-based IoT System with Secure Storage and Homomorphic Computation", IEEE Access, Vol. 14, No. 8, pp. 43472-43488, 2018.

[10] Z. Collier, M. Hassler, J. Lambert, D. DiMase, and I. Linkov, "Supply Chains", Cyber Resilience of Systems and Networks, pp. 447462, 2019.

[11] M. Queiroz, R. Telles, and S. Bonilla, "Blockchain and supply chain management 
integration: A systematic review of the literature", Supply Chain Management: An International Journal, Vol. 25, No. 2, pp. 241254, 2020.

[12] M. Abdel-Basseta, G. Manogaranb, and M. Mohameda, "Internet of Things (IoT) and its impact on supply chain: A framework for building smart, secure and efficient systems", Future Generation Computer Systems, Vol. 86, pp. 614-628, 2018.

[13] A. Sikder, G. Petracca, H. Aksu, T. Jaeger, and A. Uluagac, "A survey on sensor based threats to internet-of-things (iot) devices and applications", arXiv preprintarXiv, pp. 1802.02041, 2018.

[14] Bahga and V. Madisetti, "Blockchain platform for industrial internet of things", Journal of Software Engineering and Applications Vol. 9, No. 10, pp. 533, 2016.

[15] A. Kawa and A. Maryniak, "Blockchain Applications in Supply Chain", SMART Supply Network, pp. 21-46,2019.

[16] G. Kaladzavi, P. Diallo, C. Béré, O. Corby, I. Mirbel, M. Lo, and D. Kolyang, "Ontologiesbased Architecture for Sociocultural Knowledge Co- Construction Systems", arXiv preprint arXiv, pp. 1904.05596, 2019.

[17] S. Seebacher and M. Maleshkova, "A Modeldriven Approach for the Description of Blockchain Business Networks", In: Proc. of the 51st Hawaii International Conf. on System Sciences, pp. 3487-3496, 2018.

[18] L. Liu and W. Jia, "Business model for drug supply chain based on the internet of things", In: Proc. of Network Infrastructure and Digital Content, 2nd IEEE International Conf. on on Network Infrastructure and Digital Content, pp. 982-986, 2010.

[19] S. Altunkan, A. Yasemin, İ. Aykaç, and E. Akpinar, "Turkish pharmaceuticals track \& trace system. Health Informatics and Bioinformatics (HIBIT)", In: International Symposium on Health Informatics and Bioinformatics, pp. 2430, 2012.

[20] M. Mettler, "Blockchain technology in healthcare: The revolution starts here. e-Health Networking", In: Proc. of 18th International Conf. on E-health Networking, Applications and Services (Healthcom), pp. 1-3, 2016.

[21] B. Alangot and K. Achuthan, "Trace and Track: Enhanced Pharma Supply Chain Infrastructure to Prevent Fraud", In: Proc. of International Conf. on Ubiquitous Communications and Network Computing, pp. 189-195, 2017.
[22] T. Bocek, B. Rodrigues, T. Strasser, and B. Stiller, "Blockchains everywhere use-case of blockchains in the pharma supply-chain", In: 2017 IFIP/IEEE Symposium on Integrated Network and Service Management (IM), pp. 772-777, 2017.

[23] M. Chamekh, S. ElAsmi, M. Hamdi, and T. Kim, "Context aware middleware for RFID based pharmaceutical supply chain", In: Proc. of 13th International Wireless Communications and Mobile Computing Conf. (IWCMC), pp. 19151920, 2017.

[24] M. Wazid, A. Das, M. Khan, A. AlGhaiheb, N. Kumar, and A. Vasilakos, "Secure authentication scheme for medicine anticounterfeiting system in iot environment", IEEE Internet of Things Journal, Vol. 4, No.5, pp. 1634-1646, 2017.

[25] N. Trokanas and J. Srai, "Towards an Ontological Backbone for Pharmaceutical Digital Supply Chains", Computer Aided Chemical Engineering, Elsevier, Vol. 40, pp. 2329-2334, 2017.

[26] M. Boulos, J. Wilson, and K. Clauson, "Geospatial blockchain: promises, challenges, and scenarios in health and healthcare", International Journal of Health Geographics, pp. 17-25, 2018. 